\title{
COMPARAÇÃO DE SISTEMAS DE CULTIVO (PLANTIO DIRETO E CONVENCIONAL) E DE CULTIVARES DE TRIGO, EM SUCESSÃO À SOJA
}

\author{
Wagner Pereira Reis ${ }^{1}$, Danielle Pereira Baliza* ${ }^{1}$, Pedro Milanez de Resende ${ }^{1}$, Armando de \\ Albuquerque $^{1}$, Alexandre Martins Abdão dos Passos ${ }^{2}$, Élberis Pereira Botrel ${ }^{1}$ \\ ${ }^{1}$ Universidade Federal de Lavras/UFLA - Cx. P. 3037 - 37200-000 - Lavras, MG - * autora para correspondência \\ danibaliza@yahoo.com.br. \\ ${ }^{2}$ University of Guelph - School of Environmental Sciences - 50 Stone Road East - Guelph - N16 2W1 - Canadá.
}

\section{RESUMO}

O objetivo deste trabalho foi comparar o potencial do trigo irrigado em sistemas de plantio direto e convencional, bem como avaliar o desempenho de quinze cultivares de trigo nos dois sistemas de produção, em sucessão a soja, na região do sul de Minas Gerais. O experimento foi conduzido na Universidade Federal de Lavras (UFLA), em Lavras, MG, nos anos de 2006/07 e 2007/08, utilizou-se o delineamento em blocos casualizados em faixas com três repetições. Nas faixas foram instalados os sistemas de cultivo (convencional e plantio direto) e, nas parcelas, foram distribuídas as quinze cultivares de trigo. Foram avaliados rendimentos de grãos e dados agronômicos das plantas de trigo. $\mathrm{O}$ sistema de plantio direto proporcionou incremento médio de $25 \%$ nos teores de matéria orgânica na camada superficial do solo em relação aos teores iniciais e ao cultivo convencional indicando o potencial do sistema em melhorar a qualidade do solo. As cultivares testadas, independentes dos anos agrícolas, alteraram significativamente os rendimentos de grãos e as características agronômicas avaliadas. As altas produtividades apresentadas pelas cultivares avaliadas neste estudo, indicam o elevado potencial da cultura do trigo irrigado em sucessão a soja, para as condições clima e de solo similares ao utilizado neste estudo.

Palavras-chave: Triticum aestivum, sucessão de culturas, rendimento

\section{COMPARISON OF CROPPING SYSTEMS (NO-TILLAGE AND CONVENTIONAL) AND OF WHEAT CULTIVARS IN SUCCESSION TO THE SOYBEAN CROP}

\section{ABSTRACT}

The objective of this work was to compare the potential of the irrigated wheat under no tillage and conventional cropping systems, as well as to evaluate the performance of fifteen wheat cultivars in the two cropping systems, in succession to soybean crop, in the South of Minas Gerais. The experiment was carried out in the Federal University of Lavras (UFLA), in Lavras, MG, in the years of 2006/07 and 2007/08, in a split-block-designed scheme in a randomized complete block design, with three replications. The cropping systems (conventional and notillage) were installed in the rows and, in the columns, the fifteen wheat cultivars were distributed. The wheat yield and others agronomic characteristics were evaluated. The no-tillage provided an average increase of $25 \%$ in the organic matter levels in the topsoil layer in relation to previous levels and to conventional cropping system. The cultivars tested, independent of the agricultural year, significantly altered the grain yields and the agronomic characteristics. The high yield presented by the cultivars evaluated in this study indicates the high potential of the 
irrigated wheat cropped in succession to soybean crops, under similar climatic and soil conditions used in this study.

Key words: Triticum aestivum (L.), crops succession, yield.

\section{INTRODUÇÃO}

Práticas agrícolas que objetivam
maior sustentabilidade ambiental e econômica têm recebido crescente atenção, tanto por parte dos pesquisadores como dos agricultores. Neste contexto, o sistema de semeadura direta sem revolvimento constante do solo, o chamado plantio direto, que preconiza a sucessão e rotação de culturas têm sido bastante estudado. Tal sistema altera, sensivelmente, os componentes físicos, químicos e biológicos dos solos interferindo por sua vez na distribuição das raízes, com reflexos no crescimento da parte aérea, e consequentemente, no rendimento de grãos da espécie em cultivo (Da Ros et al. 1997, Balota et al. 1998, Franchini et al. 2000).

O plantio direto melhora a qualidade físico-química e biológica do solo em razão do aumento da matéria orgânica e da disponibilidade de nutrientes às planta (Cavalieri et al. 2004). Há uma interferência direta nos processos geoquímicos de importantes elementos como o carbono e nitrogênio resultando em menores emissões de gases do efeito estufa, com crescente seqüestro e incremento de carbono no solo, gerando ou incrementando a sustentabilidade dos sistemas (Barreto et al. 2009, Siqueira Neto et al. 2009). Neste sentido, é de fundamental importância, a utilização de espécies com alta produção de fitomassa para o desenvolvimento sustentável do plantio direto.

Outro aspecto de importância é a rotação ou sucessão de culturas, práticas essenciais que vem sendo estudadas mais detalhadamente visando o manejo alternativo e eficiente dos nutrientes como, por exemplo, a utilização de leguminosas para fornecer nitrogênio para o agroecossistema por meio da fixação biológica (Galantini et al. 2000, Martens et al. 2001, Mancin et al. 2009).

O trigo cultivado sob plantio direto tem apresentado rendimento de grãos mais elevado que sob preparo convencional do solo (Ruedell, 1995; Santos et al. 2000, 2006). No entanto, Gaertener et al. (2006) verificaram que sistemas com algum revolvimento de solo apresentaram produções de trigo mais elevadas.

Mesmo com alguns resultados contraditórios as perspectivas para o uso do sistema de plantio direto são promissoras para a cultura do trigo. Dessa forma, torna-se necessário avaliar o potencial produtivo das diversas cultivares de trigo recomendadas para o Sul de Minas Gerais no sistema de plantio direto e compará-lo ao sistema de plantio convencional, uma vez que as cultivares foram obtidas em sistema convencional (Cargnin et al. 2006, Albrecht et al. 2007), além de propiciar um melhor respaldo técnico para o manejo mais adequado da cultura do trigo em sistema de plantio direto no estado.

O presente trabalho teve por objetivo comparar o potencial do trigo irrigado em sistemas de plantio direto e convencional, bem como avaliar o desempenho de quinze cultivares de trigo nos dois sistemas de produção, em sucessão a soja, na região do sul de Minas Gerais. 


\section{MATERIAL E MÉTODOS}

O experimento foi desenvolvido em condições de campo, no município de Lavras, sul de Minas Gerais, em uma altitude média de 918 metros, a $21^{\circ} 14 \mathrm{~S}$ e $45^{\circ} 00 \mathrm{~W}$ durante os anos agrícolas de 2006/07 e 2007/08. Sendo implantado em um solo classificado como Latossolo Vermelho Distroférrico típico (LVdf)
(Embrapa, 1999). Em anos anteriores (2002 a 2003) esse solo foi cultivado em semeadura direta com trigo. No período de 2004 a 2006, a área estava sob pastagem natural sem pisoteio. Amostras desse solo das camadas de 0 a 20 e 20 a $40 \mathrm{~cm}$, foram coletadas para a realização de análises, cujas características químicas e físicas são descritas na Tabela 1.

Tabela 1. Análise química do solo nas camadas de 0 - 20cm e 20 - 40cm de profundidade, após a sucessão de culturas (soja/trigo) e preparo do solo (convencional e direto), nos anos 2006, 2007 e 2008.

\begin{tabular}{|c|c|c|c|c|c|c|c|c|c|}
\hline Tratamento & Data & $\mathrm{pH}$ & $\mathrm{P}$ & $\mathrm{K}$ & $\mathrm{Ca}$ & MG & $\mathrm{Al}$ & M.O. & $\mathrm{V}$ \\
\hline & & $\mathrm{H}_{2} \mathrm{O}$ & \multicolumn{2}{|c|}{$\mathrm{mg} \mathrm{dm}^{-3}$} & \multicolumn{3}{|c|}{$\mathrm{Cmol}_{\mathrm{c}} \mathrm{dm}^{-3}$} & $\mathrm{Dag} \mathrm{kg}^{-1}$ & $\%$ \\
\hline \multicolumn{10}{|c|}{$0-20 \mathrm{~cm}$} \\
\hline Semeadura soja & Verão/06 & 5,1 & 3,1 & 56 & 1,6 & 0,4 & 0,0 & 2,0 & 40,1 \\
\hline Pós soja Conv. & Inverno/07 & 5,5 & 3,7 & 87 & 2,3 & 0,6 & 0,0 & 2,0 & 51,8 \\
\hline Pós soja PD & Inverno/07 & 5,1 & 3,7 & 66 & 1,5 & 0,4 & 0,2 & 2,0 & 34,1 \\
\hline Pós soja Conv. & Inverno/08 & 5,2 & 11,9 & 64 & 2,4 & 0,4 & 0,1 & 2,1 & 42,5 \\
\hline Pós soja PD & Inverno/08 & 5,0 & 14,1 & 92 & 1,6 & 0,3 & 0,3 & 2,5 & 30,0 \\
\hline \multicolumn{10}{|c|}{$20-40 \mathrm{~cm}$} \\
\hline Semeadura soja & Verão/06 & 5,3 & 1,4 & 39 & 1,6 & 0,3 & 0,1 & 1,6 & 40,8 \\
\hline Pós soja Conv. & Inverno/07 & 5,5 & 1,4 & 67 & 2,0 & 0,5 & 0,0 & 1,6 & 50,7 \\
\hline Pós soja PD & Inverno/07 & 5,1 & 1,4 & 53 & 1,4 & 0,3 & 0,2 & 1,6 & 33,8 \\
\hline Pós soja Conv. & Inverno/08 & 5,5 & 3,4 & 72 & 1,9 & 0,3 & 0,1 & 2,1 & 42,7 \\
\hline Pós soja PD & Inverno/08 & 5,3 & 2,3 & 69 & 1,4 & 0,3 & 0,2 & 1,8 & 34,3 \\
\hline
\end{tabular}

$\mathrm{O}$ delineamento utilizado foi $\mathrm{em}$ blocos casualizados em faixas, com três repetições. Nas faixas foram instalados os sistemas de cultivo (convencional e plantio direto) e, nas parcelas, foram distribuídas as 15 cultivares de trigo (UFTV1 PIONEIRO, SUPERA, EMBRAPA 22, EMBRAPA 42, BRS 207, BRS 210, BRS 254, IAC 364-
TUCURUÍ III, IAC 370-ARMAGEDDON, IAC 375-PARINTINS, VI 04003, VI 04008, VI 04034, MGS BRILHANTE e IAC 350GOIAPÁ). As cultivares MGS BRILHANTE e IAC 350-GOIAPÁ são indicadas para cultivo em sequeiro. As parcelas foram constituídas por dez linhas de cinco metros de comprimento espaçadas de 
0,2 $\mathrm{m}$, sendo a parcela útil constituída pelas cinco linhas centrais.

A semeadura manual do trigo foi realizada em junho, nos dois anos de cultivo, em sucessão à soja (primavera/verão). No sistema de plantio direto o sulco foi realizado com semeadora de plantio direto e, na área de sistema convencional, foi feita a aração, gradagem e o sulcamento do solo. A adubação, controle de plantas daninhas, pragas e doenças, manejo de irrigação e demais tratos fitotécnicos foram realizados de acordo com as recomendações para o trigo (Reunião II, 2008), sendo realizadas as mesmas práticas para ambos os sistemas de produção. Na irrigação utilizou-se tensiômetros para o monitoramento da área, o volume e a frequiência das irrigações foram adotados para expectativa de produção de $5.000 \mathrm{~kg} \mathrm{ha}{ }^{-1}$, de acordo com as recomendações técnicas para a cultura do trigo. A colheita foi realizada quando os grãos apresentaram 13\% umidade.

Dados relativos às temperaturas máxima, mínima e média, à precipitação pluvial, umidade relativa e insolação no período de junho a outubro dos anos de 2007 e 2008, registrados em Lavras, são apresentados na Tabela 2.

Tabela 2. Dados relativos às temperaturas máxima (TMAX), mínima (TMIN) e média (TMED), à precipitação pluvial (PR), umidade relativa (UR) e insolação (INS) dos anos de 2007 e 2008. Lavras (MG).

\begin{tabular}{ccccccc}
\hline 2007 & TMAX & $\begin{array}{c}\text { TMIN } \\
{ }^{\circ} \mathrm{C}\end{array}$ & TMED & $\begin{array}{c}\text { PR } \\
\text { mm }\end{array}$ & $\begin{array}{c}\text { UR } \\
\%\end{array}$ & INS \\
\hline Junho & 25,8 & 11,1 & 17,3 & 5,9 & 66,3 & 263,8 \\
Julho & 25,4 & 11,1 & 17,1 & 17,6 & 66,8 & 240,0 \\
Agosto & 27,4 & 11,8 & 18,9 & 0,0 & 55,2 & 283,0 \\
Setembro & 29,8 & 14,3 & 21,3 & 0,0 & 51,2 & 278,6 \\
Outubro & 30,1 & 16,5 & 22,7 & 130,1 & 58,7 & 238,1 \\
\hline Média total & 27,7 & 13,0 & 19,4 & 30,7 & 59,6 & 260,7 \\
Acumulado & & & & 153,6 & & \\
\hline 2008 & & & & & & \\
\hline Junho & 25,1 & 12,8 & 17,6 & 14,4 & 75,8 & 204,4 \\
Julho & 25,4 & 9,9 & 16,6 & 0,0 & 61,4 & 270,9 \\
Agosto & 28,1 & 13,1 & 19,6 & 13,9 & 62,5 & 250,2 \\
Setembro & 28,0 & 13,2 & 19,4 & 87,6 & 62,1 & 238,0 \\
Outubro & 28,9 & 17,2 & 22,0 & 106,7 & 69,9 & 173,6 \\
\hline Média total & 27,1 & 13,2 & 19,0 & 44,5 & 66,3 & 227,4 \\
Acumulado & & & & 222,6 & & \\
\hline
\end{tabular}


Foram avaliadas as seguintes características na área útil, nos dois anos de cultivo e nos dois sistemas de cultivo:

- Altura de plantas - tomadas ao acaso 10 plantas por parcela, realizada no ponto de maturidade fisiológica dos grãos.

- Número de dias para o florescimento - determinação do número de dias para que as cultivares estivessem em estágio pleno de florescimento.

- Massa de mil grãos - pesagem de 1000 grãos em balança eletrônica com sensibilidade de $0,1 \mathrm{mg}$. Posteriormente, estes grãos foram reintegrados às parcelas, para obtenção do rendimento de grãos por parcela.

- Peso do hectolitro - amostra para obtenção do peso do hectolitro, que corresponde ao peso em $\mathrm{kg}$ de 100 litros de grãos. Foi retirada uma amostra do total de grãos colhidos de cada parcela. Utilizou-se o aparelho Dalle Molle, para determinação do peso do hectolitro de grãos.

- Rendimento de grãos - peso dos grãos colhidos da área útil de cada parcela. Os dados obtidos da parcela útil foram transformados em $\mathrm{kg} \mathrm{ha}^{-1}$.

As análises estatísticas foram realizadas com auxílio do software Sisvar (Ferreira, 2003), sendo aplicado o teste de Scott-Knott para comparação de médias $(p \leq 0,05)$. A dependência entre rendimento de grãos, peso do hectolitro e peso de mil grãos, no segundo ano de cultivo, foram estimadas por meio da correlação de Pearson utilizando teste de $\mathrm{t}$, com auxilio do software SAS para análise desses dados (SAS Learning Edition, 2002).

\section{RESULTADOS E DISCUSSÃO}

As análises de variância individuais para o ano de 2006/07 em relação ao rendimento de grãos, peso de mil grãos e dias para o florescimento, revelaram que a interação cultivares versus sistemas de manejo do solo apresentou variações significativas. Para peso do hectolitro apenas as cultivares diferiram entre si. E para altura das plantas, houve diferença para cultivares e sistemas de manejo de solo. Já para o ano 2007/08, a interação cultivares versus sistemas de manejo do solo influenciou o rendimento de grãos e peso do hectolitro. Para peso de mil grãos houve diferença significativa apenas para cultivares. E para dias para o florescimento verificou-se diferenças para sistemas de manejo do solo. Enquanto a altura de plantas não diferiu para nenhum dos fatores em estudo e, também para interação entre ambos.

De acordo com a análise conjunta dos resultados, o ano de cultivo e as cultivares apresentaram influência para todas as características avaliadas (Tabela 3). O efeito do ano pode ser atribuído às variações climáticas que ocorreram entre os anos de cultivo (Tabela 2), conforme verificado por Santos et al. (2000, 2006) e Guarienti et al. (2000, 2005), além de ter sido observado um ataque de pequenos pássaros na área experimental, após o período de enchimento de grão no ano de 2007/08. Já o efeito das cultivares, provavelmente foi devido as variações genéticas existentes entre essas (Cargnin et al. 2006). Para rendimento de grãos e peso do hectolitro houve diferença para interação ano versus cultivares versus sistemas de manejo do solo. Observou-se também, que as médias diferiram para altura de plantas, número de dias para o florescimento e peso de mil grãos para interação ano versus cultivares. Além disso, o número de dias para o florescimento diferiu para interação ano versus sistema de manejo de solo e anos versus cultivares. 
Tabela 3. Quadrados médios, coeficientes de variação e médias gerais para rendimento de grãos (RG), peso do hectolitro (PH), altura de plantas (AP), dias para o florescimento (DF) e peso de mil grãos (PM) do trigo, nos ensaios de sistemas de produção agrícola 2006/07 e 2007/08. Lavras, MG.

\begin{tabular}{lccccc}
\hline & \multicolumn{5}{c}{ Quadrado médio e significância } \\
\cline { 2 - 6 } & RG & PH & AP & DF & PM \\
\hline Ano (A) & $139821770,7^{*}$ & $3166,8^{*}$ & $5318,1^{*}$ & $310,4^{*}$ & $2306,2^{*}$ \\
Cultivares (C) & $7882967,4^{*}$ & $209,3^{*}$ & $684,2^{*}$ & $35,6^{*}$ & $69,6^{*}$ \\
Sistema (S) & $21442,5^{\text {n.s. }}$ & $0,1^{\text {n.s. }}$ & $1014,8^{*}$ & $0,1^{\text {n.s. }}$ & $0,6^{\text {n.s. }}$ \\
A x S & $2282356,9^{*}$ & $7,6^{\text {n.s. }}$ & $54,8^{\text {n.s. }}$ & $7,4^{*}$ & $19,8^{\text {n.s. }}$ \\
A x C & $4439457,8^{*}$ & $117,4^{*}$ & $193,2^{*}$ & $5,9^{*}$ & $47,6^{*}$ \\
S x C & $1232273,3^{*}$ & $12,8^{*}$ & $58,2^{\text {n.s. }}$ & $1,2^{\text {n.s. }}$ & $19,6^{\text {n.s. }}$ \\
A x S x C & $815800,9^{*}$ & $12,4^{*}$ & $40,0^{\text {n.s. }}$ & $2,0^{\text {n.s. }}$ & $17,6^{\text {n.s. }}$ \\
CV (\%) & 11,84 & 3,07 & 7,92 & 2,31 & 10,15 \\
Média & 2589,0 & $78,0^{*}$ & 87,6 & 69,6 & 39,3 \\
\hline
\end{tabular}

* $\mathrm{e}^{\mathrm{ns}}$ - Significativo a $5 \%$ de probabilidade, e não significativo, respectivamente.

O rendimento de grãos mais elevado ocorreu no primeiro ano de cultivo (Tabela 4). Uma provável explicação para a menor produtividade no segundo ano de cultivo, pode ser atribuído ao ataque de pequenos pássaros na área experimental no período de enchimento de grãos e início da maturação do trigo a ser colhido, o que pode ter prejudicado a produção de grão na safra 2007/08. 
Tabela 4. Efeito do ano de cultivo, de sistemas de manejo de solo (plantio direto - PD; preparo convencional - PC) e de cultivares no rendimento de grãos $\left(\mathrm{kg} \mathrm{ha}^{-1}\right)$ e peso do hectolitro $\left(\mathrm{kg} \mathrm{ha}^{-1}\right)$ do trigo. Lavras, $\mathrm{MG}^{1}$.

\begin{tabular}{|c|c|c|c|c|c|c|}
\hline & \multicolumn{3}{|c|}{ Rendimento de grãos } & \multicolumn{3}{|c|}{ Peso do hectolitro } \\
\hline & \multicolumn{6}{|c|}{ Manejo do Solo } \\
\hline & PD & $\mathrm{PC}$ & Média & PD & $\mathrm{PC}$ & Média \\
\hline \multicolumn{7}{|c|}{$2006 / 07$} \\
\hline UFVT1 Pioneiro & $2660 \mathrm{Cb}$ & $3944 \mathrm{Ba}$ & $3302 \mathrm{C}$ & $84 \mathrm{Ba}$ & $83 \mathrm{Ba}$ & $83 \mathrm{~B}$ \\
\hline Supera & $3021 \mathrm{Ba}$ & $3301 \mathrm{Ba}$ & $3161 \mathrm{C}$ & $84 \mathrm{Ba}$ & $83 \mathrm{Ba}$ & $84 \mathrm{~B}$ \\
\hline Embrapa 22 & $2878 \mathrm{Bb}$ & $3628 \mathrm{Ba}$ & $3253 \mathrm{C}$ & $83 \mathrm{Ba}$ & $83 \mathrm{Ba}$ & $83 \mathrm{~B}$ \\
\hline Embrapa42 & $3378 \mathrm{Aa}$ & $3542 \mathrm{Ba}$ & $3460 \mathrm{~B}$ & $84 \mathrm{Ba}$ & $83 \mathrm{Ba}$ & $84 \mathrm{~B}$ \\
\hline BRS 207 & $3681 \mathrm{Aa}$ & $3907 \mathrm{Ba}$ & $3794 \mathrm{~B}$ & $81 \mathrm{Ba}$ & $80 \mathrm{Ba}$ & $81 \mathrm{~B}$ \\
\hline BRS 210 & $3753 \mathrm{Aa}$ & $3508 \mathrm{Ba}$ & $3631 \mathrm{~B}$ & $80 \mathrm{Ba}$ & $81 \mathrm{Ba}$ & $81 \mathrm{~B}$ \\
\hline BRS 254 & $4059 \mathrm{Aa}$ & $4417 \mathrm{Aa}$ & $4238 \mathrm{~A}$ & $93 \mathrm{Aa}$ & $92 \mathrm{Aa}$ & $93 \mathrm{~A}$ \\
\hline IAC 364-Tucuruí III & $3323 \mathrm{Aa}$ & $3799 \mathrm{Ba}$ & $3561 \mathrm{~B}$ & $80 \mathrm{Ba}$ & $81 \mathrm{Ba}$ & $80 \mathrm{~B}$ \\
\hline IAC 370-Armageddon & $3764 \mathrm{Ab}$ & $4528 \mathrm{Aa}$ & $4146 \mathrm{~A}$ & $82 \mathrm{Ba}$ & $83 \mathrm{Ba}$ & $82 \mathrm{~B}$ \\
\hline IAC 375-Parintins & $3757 \mathrm{Aa}$ & $3702 \mathrm{Ba}$ & $3730 \mathrm{~B}$ & $84 \mathrm{Ba}$ & $83 \mathrm{Ba}$ & $84 \mathrm{~B}$ \\
\hline VI 04003 & $2872 \mathrm{Ba}$ & $2524 \mathrm{Ca}$ & $2698 \mathrm{D}$ & $83 \mathrm{Ba}$ & $81 \mathrm{Ba}$ & $82 \mathrm{~B}$ \\
\hline VI 04008 & $3660 \mathrm{Ab}$ & $4344 \mathrm{Aa}$ & $4002 \mathrm{~A}$ & $83 \mathrm{Ba}$ & $83 \mathrm{Ba}$ & $83 \mathrm{~B}$ \\
\hline VI 04034 & $4128 \mathrm{Aa}$ & $4218 \mathrm{Aa}$ & $4173 \mathrm{~A}$ & $84 \mathrm{Ba}$ & $84 \mathrm{Ba}$ & $84 \mathrm{~B}$ \\
\hline MGS Brilhante & $2396 \mathrm{Ca}$ & $1628 \mathrm{Db}$ & $2012 \mathrm{E}$ & $82 \mathrm{Ba}$ & $82 \mathrm{Ba}$ & $82 \mathrm{~B}$ \\
\hline IAC 350-Goiapá & $3201 \mathrm{Ba}$ & $2592 \mathrm{Cb}$ & $2897 \mathrm{D}$ & $83 \mathrm{Ba}$ & $82 \mathrm{Ba}$ & $82 \mathrm{~B}$ \\
\hline Média & $3369 b$ & $3572 \mathrm{a}$ & & $83 \mathrm{a}$ & $83 \mathrm{a}$ & \\
\hline Erro padrão & 176,98 & 176,98 & & 1,39 & 1,39 & \\
\hline \multicolumn{7}{|c|}{$2007 / 08$} \\
\hline UFVT1 Pioneiro & $1003 \mathrm{Db}$ & $1747 \mathrm{Ca}$ & $1375 \mathrm{D}$ & $73 \mathrm{Ba}$ & $72 \mathrm{Ca}$ & $73 \mathrm{C}$ \\
\hline Supera & $1236 \mathrm{Da}$ & $1498 \mathrm{Ca}$ & $1367 \mathrm{D}$ & $82 \mathrm{Aa}$ & $81 \mathrm{Aa}$ & $81 \mathrm{~A}$ \\
\hline Embrapa 22 & $2679 \mathrm{Bb}$ & $4082 \mathrm{Aa}$ & $3380 \mathrm{~B}$ & $80 \mathrm{Aa}$ & $82 \mathrm{Aa}$ & $81 \mathrm{~A}$ \\
\hline Embrapa42 & $2974 \mathrm{Ba}$ & $3444 \mathrm{Ba}$ & $3209 \mathrm{~B}$ & $83 \mathrm{Aa}$ & $83 \mathrm{Aa}$ & $83 \mathrm{~A}$ \\
\hline BRS 207 & $559 \mathrm{Ea}$ & $672 \mathrm{Da}$ & $616 \mathrm{~F}$ & $62 \mathrm{Db}$ & $72 \mathrm{Ca}$ & $67 \mathrm{D}$ \\
\hline BRS 210 & $1889 \mathrm{Ca}$ & $1933 \mathrm{Ca}$ & $1911 \mathrm{C}$ & $75 \mathrm{Ba}$ & $74 \mathrm{Ca}$ & $74 \mathrm{C}$ \\
\hline BRS 254 & $4853 \mathrm{Aa}$ & $4266 \mathrm{Ab}$ & $4559 \mathrm{~A}$ & $82 \mathrm{Aa}$ & $81 \mathrm{Aa}$ & $82 \mathrm{~A}$ \\
\hline IAC 364-Tucuruí III & $2806 \mathrm{Ba}$ & $736 \mathrm{Db}$ & $1771 \mathrm{C}$ & $79 \mathrm{Aa}$ & $78 \mathrm{Ba}$ & 79 B \\
\hline IAC 370-Armageddon & $788 \mathrm{Ea}$ & $1016 \mathrm{Da}$ & $902 \mathrm{E}$ & $68 \mathrm{Ca}$ & $72 \mathrm{Ca}$ & $70 \mathrm{C}$ \\
\hline IAC 375-Parintins & $585 \mathrm{Ea}$ & $381 \mathrm{Da}$ & $483 \mathrm{~F}$ & $75 \mathrm{Ba}$ & $71 \mathrm{Cb}$ & $73 \mathrm{C}$ \\
\hline VI 04003 & $549 \mathrm{Ea}$ & $279 \mathrm{Da}$ & $414 \mathrm{~F}$ & $74 \mathrm{Ba}$ & $70 \mathrm{Cb}$ & $72 \mathrm{C}$ \\
\hline VI 04008 & $1125 \mathrm{Da}$ & $575 \mathrm{Db}$ & $850 \mathrm{E}$ & $79 \mathrm{Aa}$ & $78 \mathrm{Ba}$ & $79 \mathrm{~B}$ \\
\hline VI 04034 & $4548 \mathrm{Aa}$ & $1918 \mathrm{Cb}$ & $3233 \mathrm{~B}$ & $80 \mathrm{Ab}$ & $84 \mathrm{Aa}$ & $82 \mathrm{~A}$ \\
\hline MGS Brilhante & $1556 \mathrm{Ca}$ & $653 \mathrm{Db}$ & $1105 \mathrm{D}$ & $69 \mathrm{Ca}$ & $64 \mathrm{Db}$ & $67 \mathrm{D}$ \\
\hline IAC 350-Goiapá & $318 \mathrm{Ea}$ & $503 \mathrm{Da}$ & $441 \mathrm{~F}$ & $57 \mathrm{~Eb}$ & $63 \mathrm{Da}$ & $60 \mathrm{E}$ \\
\hline Média & $1831 \mathrm{a}$ & $1584 \mathrm{a}$ & & $74 \mathrm{a}$ & $75 \mathrm{a}$ & \\
\hline
\end{tabular}

${ }^{\mathrm{T}}$ Médias seguidas pela mesma letra, maiúscula na coluna e minúscula na linha, não apresentam diferenças significativas pelo teste de Scott-Knott a $5 \%$ de probabilidade. 
Outro fator que pode ter influenciado na produção, pode ter sido o maior regime de chuvas e a elevada umidade do ar nas fases de enchimento e maturação do grão (Tabela 2). Guarienti et al. (2000, 2005), observaram que o excesso hídrico e umidade do ar elevada durante o enchimento do grãos de trigo causam redução das características físicas do grão (peso do hectolitro e peso de mil grãos) e consequentemente no rendimento desses grãos. Conforme indicações técnicas já existentes para a região, sugerimos que a semeadura do trigo não deverá entrar no mês de junho.

Condições de elevada umidade relativa e na ausência de vento, o processo de difusão é lento e a perda de água é pequena. Quando os estômatos estão fechados, a transpiração diminui sensivelmente, podendo até tornar-se desprezível. Em condições isotérmicas, de uma atmosfera saturada de água, quando houver precipitação pluvial e a umidade relativa for elevada, e de uma planta túrgida, não há fluxo de água na planta. Não ocorrendo fluxo de água na planta e, consequientemente, de nutrientes, há redução de produtos fotossintéticos, como carboidratos, lipídios, aminoácidos e outros, responsáveis pelo enchimento de grãos. Isso poderia explicar em parte os resultados encontrados indicando que, de acordo com esse mecanismo, os grãos tornar-se-iam menores, de reduzidos peso de mil grãos e peso do hectolitro e, conseqüientemente, afetariam o rendimento de grãos.

No primeiro ano de cultivo os rendimentos dos grãos de trigo no sistema convencional, superaram o plantio direto (Tabela 4) em $203 \mathrm{~kg} / \mathrm{ha}$, com o plantio de trigo efetuado em sucessão a soja. Este resultado corrobora ao obtido por Gaertener et al. (2006), ao desenvolverem estudo com sistemas de manejo de solo, verificaram que sistemas com algum revolvimento de solo apresentaram produções de trigo mais elevadas. Contudo, discorda dos trabalhos de Santos et al. (2000, 2006) no qual o rendimento de grãos mais elevado ocorreu no sistema de plantio direto e no cultivo mínimo, em comparação com preparos convencionais de solo. Para esses autores, as diferenças foram atribuídas à deficiência hídrica em alguma fase crítica de desenvolvimento da cultura e ao acúmulo de nutrientes e de matéria orgânica na camada superficial no solo.

Deve ser enfatizado, que o presente trabalho foi conduzido sob irrigação, dessa forma, não houve deficiência hídrica durante o desenvolvimento da cultura. Diferentemente, do observado nos trabalhos de Santos et al. (2000, 2006), onde a precipitação pluvial foi baixa e mal distribuída durante o decorrer dos anos. Com relação ao acúmulo de nutrientes e matéria orgânica na camada superficial do solo, verifica-se no primeiro ano de cultivo que não houve maior acúmulo no plantio direto quando comparado ao sistema convencional de preparo do solo (Tabela 1). Isto, provavelmente contribuiu para não superioridade do sistema de plantio direto.

Independente do sistema de manejo do solo adotado, foi observado um elevado potencial de produção de trigo irrigado no período outono/inverno nas condições edafoclimáticas deste estudo, com produtividades expressivas, acima de quatro toneladas por hectare.

Para o segundo ano de cultivo, os rendimentos dos grãos de trigo não diferiram entre si para ambos os sistemas de manejo de solo (Tabela 7). Verifica-se que o sistema de plantio direto torna-se mais eficiente para o trigo irrigado a medida que o sistema vai sendo implantado no campo, em sucessão com a soja na primavera/verão. Este fato, pode ser exemplificado pelo aumento em $25 \%$ no acúmulo de matéria orgânica na camada superficial do solo no sistema de 
plantio direto no período de um ano (Tabela 1). De acordo com Cavalieri et al. (2004), o plantio direto melhora a qualidade físicoquímica e biológica do solo, em razão do aumento da matéria orgânica e da disponibilidade de nutrientes às planta.

As cultivares de trigo com maior rendimento de grãos no sistema convencional, nos dois anos de cultivo, foram a UFVT1 Pioneiro e Embrapa 22. No sistema de plantio direto, as cultivares mais produtivas para o primeiro ano de cultivo foram: Embrapa 42, BRS 207, BRS 210, BRS 254, IAC 364-Tucuruí III, IAC 370Armageddon, IAC 375-Parintins, VI 04008 e VI 04034 e as cultivares BRS 254 e VI 04034 para o segundo ano. Tal fato, demonstra a importância em estudar e avaliar a adaptabilidade das cultivares em diferentes sistemas de cultivo e manejo do solo. Albrecht et al. (2007), verificaram a adaptabilidade de genótipos de trigo irrigado em diferentes locais e Cargnin et al. (2006), em diferentes épocas de semeadura. Contudo, esses autores não avaliaram a adaptação desses genótipos em diferentes sistemas de manejo do solo.

Em 2006/07, a cultivar BRS 254 apresentou a maior média de rendimento de grãos (4238 kg ha-1), não diferindo das cultivares: IAC 370-Armageddon, MGS Brilhante e VI040034. Em 2007/08, a cultivar BRS 254 foi superior as demais cultivares. A BRS 254, proveniente do cruzamento da Embrapa 22 com a cultivar Anahuac, recomendada para o estado de Minas Gerais sob cultivo irrigado, possui potencial de produtividade de 6 toneladas por hectare, além de alta força de glúten e estabilidade, características que as indústrias exigem para a panificação (Reunião III, 2009).

O peso do hectolitro é uma das características que determina a qualidade industrial do trigo (Guarienti et al. 2000,
2005). O peso do hectolitro mais elevado ocorreu no primeiro ano de cultivo (Tabela 4) onde foi obtido um valor médio acima do padrão nacional (83), o que não ocorreu em 2007/08 (75) demonstrando uma menor densidade dos grãos, devido possivelmente à uma maior taxa de respiração gerada por estresses como excesso de chuva e o ataque de pássaros, já discutidos anteriormente. De acordo com Bhatt et al. (1981), a redução do peso do hectolitro é resultante da alta taxa de respiração, associada aos grãos germinados, que consomem carboidratos acumulados nos grãos.

Para o peso do hectolitro nos dois anos de cultivo, não houve diferença significativa entre os sistemas de manejo do solo. Guarienti et al. (2000), também não observaram diferenças significativas no peso do hectolitro em relação aos sistemas de manejo de solo (plantio direto, preparo convencional de solo com arado de discos, com arado de aivecas e cultivo mínimo). Já Santos et al. (2006) verificaram que os sistemas conservacionistas (plantio direto e cultivo mínimo) favoreceram o peso do hectolitro e a altura de plantas de trigo mais elevadas do que o preparo convencional do solo. Dessa forma, verifica-se que as pesquisas com sistemas de manejo de solo para o trigo, apresentam resultados ainda contraditórios, sendo necessários mais estudos.

No ano de 2006/07 a cultivar BRS 254 foi superior às outras cultivares para peso do hectolitro em ambos os sistemas de manejo do solo. Já no ano de 2007/08 destacaram-se as cultivares: Supera, Embrapa 22, Embrapa 42, BRS 254, VI04034 nos sistemas de plantio direto e convencional. No sistema de plantio direto as cultivares IAC 364-Tucuruí III e VI04008 não diferiram das superiores.

Os dados de massa de mil grãos da mesma forma que os dados de peso do 
hectolitro concordam com a menor produtividade no segundo ano de cultivo com o trigo, sendo que neste ano condições adversas já citadas provavelmente podem explicar estas reduções (Tabela 5). A correlação entre peso do hectolitro e rendimento de grãos no ano de 2007/08 foi positiva $(62,91 \%)$ e significativa $(<0,0001)$. $\mathrm{O}$ mesmo foi verificado entre massa de mil grãos e rendimento de grãos com uma correlação positiva $(37,66 \%)$ e significativa $(<0,0003)$.

Tabela 5. Efeito do ano de cultivo e de cultivares na massa de mil grãos do trigo (PM). Lavras,

\begin{tabular}{lccc}
\multicolumn{1}{c}{ MG $^{1}$ Cultivares } & \multicolumn{3}{c}{} \\
\cline { 2 - 4 } & $2006 / 07$ & $2007 / 08$ & Média \\
\hline UFVT1 Pioneiro & $43 \mathrm{Aa}$ & $39 \mathrm{Ba}$ & $41 \mathrm{~A}$ \\
Supera & $40 \mathrm{Aa}$ & $33 \mathrm{Cb}$ & $36 \mathrm{~B}$ \\
Embrapa22 & $43 \mathrm{Aa}$ & $37 \mathrm{Bb}$ & $40 \mathrm{~A}$ \\
Embrapa42 & $44 \mathrm{Aa}$ & $41 \mathrm{Aa}$ & $42 \mathrm{~A}$ \\
BRS 207 & $42 \mathrm{Aa}$ & $34 \mathrm{Cb}$ & $38 \mathrm{~B}$ \\
BRS 210 & $40 \mathrm{Aa}$ & $34 \mathrm{Cb}$ & $37 \mathrm{~B}$ \\
BRS 254 & $42 \mathrm{Aa}$ & $39 \mathrm{Ba}$ & $40 \mathrm{~A}$ \\
IAC 364-Tucuruí III & $43 \mathrm{Aa}$ & $37 \mathrm{Bb}$ & $40 \mathrm{~A}$ \\
IAC 370-Armageddon & $46 \mathrm{Aa}$ & $36 \mathrm{Bb}$ & $41 \mathrm{~A}$ \\
IAC 375-Parintins & $40 \mathrm{Aa}$ & $30 \mathrm{Db}$ & $35 \mathrm{~B}$ \\
VI 04003 & $45 \mathrm{Aa}$ & $37 \mathrm{Bb}$ & $41 \mathrm{~A}$ \\
VI 04008 & $44 \mathrm{Aa}$ & $36 \mathrm{Bb}$ & $40 \mathrm{~A}$ \\
VI 04034 & $44 \mathrm{Aa}$ & $44 \mathrm{Aa}$ & $44 \mathrm{~A}$ \\
MGS Brilhante & $43 \mathrm{Aa}$ & $35 \mathrm{Cb}$ & $39 \mathrm{~A}$ \\
IAC 350-Goiapá & $46 \mathrm{Aa}$ & $28 \mathrm{Db}$ & $37 \mathrm{~B}$ \\
Média & $43 \mathrm{a}$ & $35 \mathrm{~b}$ & \\
\hline
\end{tabular}

${ }^{1}$ Médias seguidas pela mesma letra, maiúscula na coluna e minúscula na linha, não apresentam diferenças significativas pelo teste de Scott-Knott a $5 \%$ de probabilidade.

No ano de 2006/07, as cultivares não diferiram entre si para a masa de mil grãos. Já para o ano de 2007/08, a Embrapa 42 e VI04034 foram superiores às demais (Tabela 5). Sabe-se que a cultivar Embrapa 42 é recomendada para cultivo sob irrigação no estado de Minas Gerais (Reunião III, 2009).

Foi observada diferença entre os anos de cultivo para o florescimento (Tabela 6). No ano de 2006/07, as cultivares floresceram em média quatro dias antes do que no ano de 2007/08. No entanto, se considerar os valores médios para os dois anos de cultivo, o florescimento das cultivares se deu próximo aos 70 dias após a emergência das plantas. Sob ponto de vista de cultivo, o florescimento ocorrendo fora do período de ocorrência de geadas, a cultura não tem o risco de apresentar problemas relacionados ao fenômeno climático, ou seja, esterilidade do grão de pólen. Assim, com a semeadura no final de maio o florescimento das cultivares avaliadas se deu em meados de agosto, fora do período de provável risco de ocorrência de geadas, que é na segunda semana de junho e primeira de julho. 
Tabela 6. Efeito do ano de cultivo e de cultivares no número de dias para o florescimento (DF) e altura de plantas (AP) de trigo. Lavras, $\mathrm{MG}^{1}$.

\begin{tabular}{lcccccc}
\hline \multicolumn{1}{c}{ Cultivares } & \multicolumn{3}{c}{$\mathrm{DF}$} & $\mathrm{AP}$ & \\
\cline { 2 - 6 } & $06 / 07$ & $07 / 08$ & Média & $06 / 07$ & $07 / 08$ & Média \\
\hline UFVT1 Pioneiro & $66 \mathrm{Cb}$ & $72 \mathrm{Aa}$ & $69 \mathrm{~B}$ & $85 \mathrm{Cb}$ & $96 \mathrm{Ba}$ & $91 \mathrm{~B}$ \\
Supera & $65 \mathrm{Cb}$ & $72 \mathrm{Aa}$ & $68 \mathrm{C}$ & $83 \mathrm{Ca}$ & $90 \mathrm{Ba}$ & $86 \mathrm{C}$ \\
Embrapa22 & $68 \mathrm{Bb}$ & $72 \mathrm{Aa}$ & $70 \mathrm{~B}$ & $82 \mathrm{Cb}$ & $91 \mathrm{Ba}$ & $87 \mathrm{C}$ \\
Embrapa42 & $68 \mathrm{Bb}$ & $72 \mathrm{Aa}$ & $70 \mathrm{~B}$ & $91 \mathrm{Ba}$ & $90 \mathrm{Ba}$ & $90 \mathrm{~B}$ \\
BRS 207 & $70 \mathrm{Ab}$ & $72 \mathrm{Aa}$ & $71 \mathrm{~A}$ & $75 \mathrm{Db}$ & $91 \mathrm{Ba}$ & $83 \mathrm{C}$ \\
BRS 210 & $70 \mathrm{Aa}$ & $71 \mathrm{Aa}$ & $71 \mathrm{~B}$ & $69 \mathrm{Db}$ & $87 \mathrm{Ba}$ & $78 \mathrm{C}$ \\
BRS 254 & $68 \mathrm{Bb}$ & $72 \mathrm{Aa}$ & $70 \mathrm{~B}$ & $79 \mathrm{Cb}$ & $91 \mathrm{Ba}$ & $85 \mathrm{C}$ \\
IAC 364-Tucuruí III & $68 \mathrm{Bb}$ & $72 \mathrm{Aa}$ & $70 \mathrm{~B}$ & $83 \mathrm{Cb}$ & $95 \mathrm{Ba}$ & $89 \mathrm{~B}$ \\
IAC 370-Armageddon & $70 \mathrm{Ab}$ & $72 \mathrm{Aa}$ & $71 \mathrm{~A}$ & $77 \mathrm{Db}$ & $94 \mathrm{Ba}$ & $85 \mathrm{C}$ \\
IAC 375-Parintins & $70 \mathrm{Aa}$ & $72 \mathrm{Aa}$ & $71 \mathrm{~A}$ & $79 \mathrm{Cb}$ & $94 \mathrm{Ba}$ & $86 \mathrm{C}$ \\
VI 04003 & $62 \mathrm{Db}$ & $70 \mathrm{Aa}$ & $66 \mathrm{D}$ & $77 \mathrm{Db}$ & $91 \mathrm{Ba}$ & $84 \mathrm{C}$ \\
VI 04008 & $64 \mathrm{Cb}$ & $71 \mathrm{Aa}$ & $68 \mathrm{C}$ & $74 \mathrm{Db}$ & $92 \mathrm{Ba}$ & $83 \mathrm{C}$ \\
VI 04034 & $65 \mathrm{Cb}$ & $71 \mathrm{Aa}$ & $68 \mathrm{C}$ & $73 \mathrm{Db}$ & $93 \mathrm{Ba}$ & $83 \mathrm{C}$ \\
MGS Brilhante & $71 \mathrm{Ab}$ & $74 \mathrm{Aa}$ & $72 \mathrm{~A}$ & $114 \mathrm{Aa}$ & $108 \mathrm{Aa}$ & $111 \mathrm{~A}$ \\
IAC 350-Goiapá & $71 \mathrm{Ab}$ & $74 \mathrm{Aa}$ & $72 \mathrm{~A}$ & $94 \mathrm{Ba}$ & $93 \mathrm{Ba}$ & $94 \mathrm{~B}$ \\
Média & $68 \mathrm{~b}$ & $72 \mathrm{a}$ & & $82 \mathrm{~b}$ & $93 \mathrm{a}$ & \\
\hline
\end{tabular}

${ }^{\mathrm{T}}$ Médias seguidas pela mesma letra, maiúscula na coluna e minúscula na linha, não apresentam diferenças significativas pelo teste de Scott-Knott a $5 \%$ de probabilidade.

O florescimento das cultivares variou no primeiro ano de cultivo, com variação de nove dias, sendo mais precoce a VI 04003. Este resultado indica que o programa de melhoramento da UFV/EPAMIG, tem sido eficiente na obtenção de genótipo de ciclo mais curto. Cargnin et al. (2006), na região Coimbra-MG no ano de 2004, observaram que os diferentes genótipos exibem florescimento diferenciado. No ano de 2007/08, as cultivares não diferiram entre si.

A variação nos anos de cultivo apresentou influência no crescimento em altura das plantas de trigo (Tabela 6). Foi observada altura mais elevada das plantas no segundo ano de cultivo, diferente do observado para rendimento de grãos, peso do hectolitro e peso de mil grãos que apresentaram menores valores no ano de 2007/08. O maior crescimento em altura da planta pode ser explicado pela melhoria nas características físicas e químicas do solo, a medida que a sucessão da soja e trigo foi implantada.

Para os dois anos de cultivo, a cultivar MGS Brilhante apresentou a maior altura e foi superior às outras cultivares. Cargnin et al. (2006), também verificou resposta diferenciada dos genótipos para altura das plantas.

No florescimento, houve diferenças significativas entre sistemas de manejo do solo versus ano de cultivo (Tabela 7). Com relação aos valores médios, os sistemas de manejo do solo não diferiram entre si. Verifica-se no ano de 2006/07, que o florescimento das plantas de trigo foi mais precoce, no entanto, como discutido anteriormente em nenhum dos dois anos de cultivo não houve riscos de geada. 
Tabela 7. Efeito do ano de cultivo e de sistemas de manejo de solo (plantio direto - PD; preparo convencional - PC) no número de dias para o florescimento (DF) do trigo. Lavras, $\mathrm{MG}^{1}$.

\begin{tabular}{cccc}
\hline Ano de cultivo & \multicolumn{3}{c}{ DF } \\
\cline { 2 - 4 } & PD & PC & Média \\
\hline $2006 / 07$ & $68 \mathrm{Ba}$ & $67 \mathrm{Ba}$ & $68 \mathrm{~B}$ \\
$2007 / 08$ & $71 \mathrm{Ab}$ & $72 \mathrm{Aa}$ & $72 \mathrm{~A}$ \\
Média & $70 \mathrm{a}$ & $70 \mathrm{a}$ & \\
\hline
\end{tabular}

${ }^{T}$ Médias seguidas pela mesma letra, maiúscula na coluna e minúscula na linha, não apresentam diferenças significativas pelo teste de Scott-Knott a $5 \%$ de probabilidade.

\section{CONCLUSÕES}

O sistema de plantio direto proporcionou incremento médio de $25 \%$ nos teores de matéria orgânica na camada superficial do solo em relação aos teores iniciais e ao cultivo convencional indicando o potencial do sistema em melhorar a qualidade do solo.

As cultivares testadas, independentes dos anos agrícolas, alteraram significativamente os rendimentos de grãos e as características agronômicas avaliadas.

$\mathrm{O}$ trigo irrigado, cultivado em sucessão a soja, apresenta elevado potencial produtivo, para região do sul de Minas Gerais.

\section{REFERÊNCIAS BIBLIOGRÁFICAS}

ALBRECHT, J.C.; VIEIRA, E.A.; SILVA, M.S.; ANDRADE, J.M.V. de; SCHEEREN, P.L.; TRINDADE, M.G.da; SOARES SOBRINHO, J.; SOUSA, C.N.A. de; REIS, W.P.; RIBEIRO JÚNIOR, W.Q.; FRONZA, V.; CARGNIN, A.; YAMANAKA, C.H. 2007. Adaptabilidade e estabilidade de genótipos de trigo irrigado no cerrado do Brasil Central. Pesquisa Agropecuária Brasileira, v.42, p.1727-1734.
BHATT, G.M.; PAULSEN, G.M.; KULP, K.; HEYNE, E. 1981. Preharvest sprouting in hard winter wheats: assessment of methods to detect genotypic and nitrogen effects and interactions. Cereal Chemistry, v.58, p.300-302.

BALOTA, E.L.; COLOZZI FILHO, A.; ANDRADE, D.S.; HUNGRIA, M. 1998. Biomassa microbiana e sua atividade em solos sob diferentes sistemas de preparo e sucessão de culturas. Revista Brasileira de Ciência do Solo, v.22, p.641-649.

BARRETO, R.C.; MADARI, B.E.; MADDOCK, J.E.L.; MACHADO, P.L.O.A.; TORRES, E.; FRANCHINI, J.; COSTA, A. R. 2009. The impact of soil management on aggregation, carbon stabilization and carbon loss as $\mathrm{CO} 2$ in the surface layer of a Rhodic Ferralsol in Southern Brazil . Agriculture, Ecosystems \& Environment. v.132, p.243-251.

CARGNIN, A.; SOUZA, M.A. de; CARNEIRO, P.C.S.; SOFIATTI, V. 2006. Interação entre genótipos e ambientes e implicações em ganhos com seleção em trigo. Pesquisa Agropecuária Brasileira, v.41, p.987993.

CAVALIERI, K. M. V.; TORMENA, C. A.; FIDALSKI， J.; PINTRO, J. C.; 
COSTA, A. C. S.; SOUZA JUNIOR, I. G. 2004. Alterações nas propriedades químicas de um LATOSSOLO VERMELHO Eutroférrico por dois sistemas de manejo de solo. Acta Scientiarum. Agronomy, v. 26, p. 377385.

DA ROS, C.O.; SECCO, D.; FIORIN, J.E.; PETRERE, C.; CADORE, M.A.; PASA, L. 1997. Manejo do solo a partir de campo nativo: efeito sobre a forma e estabilidade da estrutura ao final de cinco anos. Revista Brasileira de Ciência do Solo, v.21, p.241-247.

EMBRAPA. 1999. Centro Nacional de Pesquisa de Solos. Sistema brasileiro de classificação de solos. Rio de Janeiro: Embrapa solos, 412p.

FRANCHINI, J.C.; BORKERT, C.M.; FERREIRA, M.M.; GAUDÊNCIO, C.A. 2000. Alterações na fertilidade do solo em sistemas de rotação de culturas em semeadura direta. Revista Brasileira de Ciência do Solo, v.24, p.459-467.

FERREIRA, D.F. Sisvar. 2003 - Sistema para análise de variância de dados balanceados: programa de análises estatísticas e planejamento de experimentos, versão 4. Lavras: UFLA.

GAERTNER， C.; DEDECEK， R.A.; BISCAIA, R.M. 2006. Produtividade do trigo e da soja em latossolo vermelho distrófico com diferentes níveis de erosão hídrica. Scientia Agrária, v.7, p.27-34.

GALANTINI, J. A.; LANDRISCINI, M. R.; IGLESIAS, J. O.; MIGLIERINA, A. M.; ROSSELL, R. A. 2000. The effects of crop rotation and fertilization on wheat productivity in the Pampean semiarid region of Argentina. 2. Nutrient balance, yield and grain quality. Soil \& Tillage Research., v. 53, p. 137-144.
GUARIENTI, E.M.; CIACOO, C.F.; CUNHA, G.R. da; DEL DUCA, L.J.A.; CAMARGO, C.M.O. de. 2005. Efeitos da precipitação pluvial, da umidade relativa do ar e de excesso e déficit hídrico do solo no peso do hectolitro, no peso de mil grãos e no rendimento de grãos de trigo. Ciência Tecnologia de Alimentos, v.25, p.412-418.

GUARIENTI, E.M.; SANTOS, H.P. dos; LHAMBY, J.C.B. 2000. Influência do manejo do solo e da rotação de culturas na qualidade industrial do trigo. Pesquisa Agropecuária Brasileira, v.35, p.2375-2382.

MANCIN, C.R.; SOUZA, L.C.F. de; NOVELINO, J.O.; MARCHETTI, M.E.; GONÇALVES, M.C. 2009. Desempenho agronômico da soja sob diferentes rotações e sucessões de culturas em sistema de plantio direto. Acta Scientiarum. Agronomy, v.31, p.71-77.

MARTENS, J. R. T.; HOEPPNER, J. W.; ENTZ, M. H. 2001. Legume cover crops with winter cereals in southern Manitoba: establishment, productivity, and microclimate effects. Agronomy Journal, v. 93, p. 1086-1096.

REUNIÃO DA COMISSÃO BRASILEIRA DE PESQUISA DE TRIGO E TRITICALE II, 2008. Passo Fundo. Informações técnicas para a safra 2009: trigo e triticale. Passo Fundo: Embrapa-CNPT, 172p.

REUNIÃO DA COMISSÃO BRASILEIRA DE PESQUISA DE TRIGO E TRITICALE III, 2009. Veranópolis. Informações técnicas para a safra 2010: trigo e triticale. Veranópolis: Embrapa-CNPT, 172p.

RUEDELL, J. 1995. Plantio direto na região de Cruz Alta. Cruz Alta: FUNDACEP FECOTRIGO, 134p.

SAS LEARNING EDITION. 2002. Programa SAS - getting started with 
the SAS Learning Edition. Cary: SAS Publishing, 200p.

SANTOS, H.P.; LHAMBY, J.C.B.; PRESTES, A.M.; LIMA, M.R. 2000. Efeito de sistemas de manejo de solo e de rotação de culturas de inverno no rendimento e doenças de trigo. Pesquisa Agropecuária Brasileira, v.35, p.2355-2361.

SANTOS, H. P. dos; LHAMBY, J. C. B.; SPERA, S. T.; ÁVILA, A. 2006. Efeito de práticas culturais sobre o rendimento e outras características agronômicas do trigo. Bragantia, v. 65, p.669-677.

SIQUEIRA NETO, M.; PICCOLO, M.C. de; FEIGL, B.J.; VENZKE FILHO, S.P. de; CERRI, C.E.P.; CERRI, C.C. 2009. Rotação de culturas no sistema plantio direto em Tibagi (PR): II - Emissões de $\mathrm{CO} 2$ e N2O. Revista Brasileira de Ciência do Solo, v.33, p. 1023-1029. 